\title{
The role of emotion, vision and touch in movement learning neuroplasticity and the mirror neuron system
}

\begin{abstract}
The brains ability to restructure, linked with positive emotional states and techniques to enhance the activation of the mirror neuron system, allows for increased motor learning. This study examines the effects of enhancing goal-oriented movement and examines various techniques used to strengthen neural networks during such movement practices. Goal oriented movement practices are commonly used when engaging in Pilates. Participants will be divided between three groups to help identify potential differences between individuals who receive sensory cueing and those who do not receive sensory cueing. It is hypothesized there will be a difference between participants who receive emotional priming and the visual enhancement of touch versus those who are not primed. Specifically, it is predicted that participants who are primed will demonstrate enhanced skill acquisition of learning during Pilates as opposed to participants assigned to either the regular Pilates condition or the control condition. These types of studies are important in that they can possibly be applied in many motor learning areas, such as physical therapy, sports, general physical activity for health, and possibly even psychological interventions. Results of the current study supported the main hypothesis in that increased physical task skill was obtained in the group receiving positive emotional cueing and visual enhancement of touch. Likewise there was a statically significant increase in physical self-efficacy scores in the group receiving these independent variables over that of the group receiving regular Pilates only. The results of the current study show that a physical task environment such as that in a Pilates class can be improved by adding techniques that enhance sensory input to the regions of the brain that recognize and process motor activity. Also that with an increase in physical skill comes a heightened physical self-efficacy.
\end{abstract}

In theory this was due to an enhancement of receptivity in the regions of the brain responsible for motor leaning which includes the mirror neuron system, as well as the emotional centers of the brain which are responsible for goal motivation and self-efficacy.

Keywords: neuroplasticity, somatosensory, mirror neuron system, pilates techniques, visual enhancement, electromyogram
Volume 3 Issue 5 - 2015

\author{
Jeanne Masterson \\ Department of Psychology, Dominican University of California, \\ USA
}

Correspondence: Jeanne Masterson, Department of Psychology, Dominican University of California, 50 Acacia Avenue San Rafael, Ca. 9490I, USA, Tel 41 5-342-3083, Email Jeannemasterson@alumni,Dominican.edu

Received: September 23, 2015 | Published: September 24, 2015
Abbreviations: PPA, perceived physical ability physical; PSPC, self-presentation confidence; PSE, physical self-efficacy scale; fMRI, functional magnetic resonance imaging; TMS, transcranial magnetic stimulation; EEG, electroencephalogram; PS-ES, physical self-efficacy scale; MSS, the mood survey scale; LM, level of mood; $\mathrm{RS}$, reactivity to situations

\section{Introduction}

Neuroplasticity is the brains ability to form new neural connections in response to new situations or changes in the environment. Motor learning is improved during activation of the mirror neuron system, in which regions of the brain are accessed and neural receptivity heightened using various sensory enhancement techniques. The activation of the mirror neuron system is correlated with improvements in physical skills as well as motor learning. The areas of the brain that are involved in motor learning include pre-motor, motor cortex and somatosensory cortex, as well as a number of additional cortices. According to a study by Gallese et al., ${ }^{1}$ the premotor cortex, the motor cortex, the somatosensory cortex are responsible for motor perception and action. ${ }^{1}$ However, as demonstrated in their study, there also appears to be a link between motor learning and the emotional centers of the brain. ${ }^{1}$ This link is the insular cortex, which can be described as a bridge between the motion or action areas of the brain and the parts of the brain that assign meaning to an action. In a study conducted by Immordino-Yang ${ }^{2}$ emotion is referred to as 'smoke' around mirror neurons. Immordino-Yang labels emotions as potential action or inhibitory factors in skill acquisition. In Immordino's report, perceptions constructed by emotions can create a cognitive construct that may either enhance or inhibit a goal oriented learning, such as skill acquisition. ${ }^{2}$ As described in Immordino-Yang's report, this skill can be perceived in the mirror neuron system via the sensory organs that are shaped by the contextual perception from either previous experiences or lack of, including emotion. Immordino-Yang states that this information is important as in that external factors such as emotional affect, can alter the effectiveness of a learning environment by either strengthening the understanding of a goal or weakening it, as emotional states can affect the desire of one to reach their goals. This statement brings to light the importance of setting emotional states to enhance learning, not inhibit the goal.

To better define terms used throughout this study, motor learning is the process of improving motor skills through repeated application, or replication of a movement pattern. Mirror neurons are specific neural structures in the brain that fire when an observer views a motor action of another. For example: when one observes another bringing food to their mouth, neurons fire in the observers brain similar to those that are firing in the one doing the action. They are considered a 
mechanism for the perception of action of movement and for imitation of action, as well as interpretation of emotional facial expression and language acquisition. Emotional priming is an act whereby a verbal representation of an emotion is given prior to a task. For example: 'Doing puzzles is good', would be a positive emotional prime for someone about to be asked to complete a puzzle task. It assists in setting an emotional tone prior to an act. ${ }^{3}$ Visual enhancement of touch is defined as an enhancement to sensory input by combining two sensory stimuli prior to a motor task. It is used to enhance motor learning as a combination of sensory stimuli can strengthen the neural response to the areas of the brain responsible for movement. In this study vision and touch are combined as they both assist in activation of the regions of the brain responsible for motor perception and action.

To best measure the effectiveness of motor learning theories, are measures that rate the physical self-efficacy or confidence in carrying out a physical task and the competency of effectively completing the task. Studies on reliability and validity of these measures have been carried out with success when applied to competitive sports settings. In a study by McAuley and Gill ${ }^{4}$ the researchers put to test the application of Ryckman, et al., ${ }^{5}$ Perceived Self-Efficacy (PSE) measure, and further analyzed its two sub scales reflecting the participants Perceived Physical Ability (PPA) and Physical Self-Presentation Confidence (PSPC). The McAuley and Gill study ${ }^{4}$ examined the role of selfconfidence and its correlation to sports performance. In their study the researchers recruited 52 female college gymnastic athletes from 7 universities. ${ }^{5}$ The participants were asked to complete an inventory assessing their confidence before the performance, this was done prior to their event warm-ups. The gymnasts were ask to rate on the Physical Self-Efficacy scale, how they perceived they would perform in 4 task specific events; the vault, bars, balance beam and floor exercise. The -gymnasts were asked in each of 4 events contained 7 specific maneuvers. e.g. The vault event consisted of a handspring, half on half off, handspring full twist, tsukahara (tuck or pike), layout tsukahara, handspring front. Of these 7 maneuvers in the 4 events, the gymnasts were asked to indicate how many they thought they could successfully complete. The gymnasts were also asked how confident they were that they could complete each maneuver. The gymnasts were additionally asked to predict their actual score. At the conclusion of the gymnastic meet the director of the event supplied the researcher with a copy of the official score sheets.

The data from the measures was analyzed in several factors. Perceived Physical Ability (PPA) and Physical Self-Presentation Confidence (PSPC) dimensions were examined. Measures were tested and analyzed for reliability and validity in their application to a sport setting. The results relayed that the Physical Self-Efficacy Scale (PSE) appeared to be significantly reliable, and a valid measure of general physical self-efficacy, though the PSE was not found to be a significant predictor of sports performance. Yet a combination of PSE and task specific self-efficacy and predictions of performance, were significant predictors of actual performance.

\section{Motor Learning And Mirror Neuron System}

Previous studies reveal that people learn physical behavior efficiently when watching or imitating a movement. ${ }^{6}$ According to a report by Bastiaansen et al., ${ }^{7}$ the mirror neuron system has three primary roles; first to help us understand what another person is performing and how they are performing it, so we can better recognize the goal of the action. ${ }^{7}$ Second, it is responsible for imitation of an action, and is more strongly activated during the actual imitation than of the observation prior. The third role the mirror neuron systems concerns allowing us to empathize, and have an intuition of the emotions of others, while also sharing in their emotional states. In addition to the three duties the mirror neuron system is also involved in neural circuits for sensory perceptions, such as vision and touch.

The authors refer to a study by ${ }^{8}$ where by subjects where shown movies of someone else's leg being touched by a stick. Later the same subjects were touched on their own legs. Activity in the somatosensory regions of the brain were measured using Functional Magnetic Resonance Imaging (fMRI) during both events, and when the subjects viewed someone else's leg being touched, the same regions of the brain were activated similar to when their own leg was being touched, though the activation was not as strong, suggesting a relation between viewing touch on another, and being touched in a similar fashion. The perceptions of both events activated regions of the mirror neuron system and this activation was strengthened when using a combination of vision and touch with the subject.

The study consisted of three experimental conditions. In the first experiment, participants were made up of six females and eight males, ages ranging from 22-28years of age. In this condition, the stimuli and conditions consisted of four functional runs, containing two tactile and two visual stimulations. In the tactile runs the participants were instructed to keep their eyes closed, the experimenter entered the room and stimulated the anterior lower portion of the participants leg, using and up and down brushing motion. This was repeated on each leg in a 24 second block, with a rest block of an equal 24 seconds.

The second experiment consisted of eight participants. In the second condition, the participants were exposed to four blocks with the first three being visual and the last tactile. The visual runs were arranged in the order of increasing touch, which progressed from stimuli not related to touch, to legs of another being touched in the film the participants were watching, to the participants' leg being touched. Each of the visual runs lasted 648seconds, and only differed by the visual stimuli seen by the participant. One visual stimuli in the first condition was of airplane wings moving up and down over an island, there was no impression of touch given in this run. In run two a film containing objects that represented legs, with the same objects touching the legs in the film as that of the first run.

In the third experiment, there were 7 participants. During the third run, the movies of the first study were used and the visual representation was that of others legs, both a man and a woman. Runs three and four were replications of the first study. In run four, the length of the tactile stimulation was reduced to 12 seconds. Following analysis, results revealed that the secondary somatosensory regions of the brain were activated both when the participants observed someone and something being touched by objects, as well as when they were touched themselves. The results reported that the primary somatosensory regions of the brain showed activation during the observation of touch, yet it was substantially weaker.

Motor skills involve perception as well as action. By enhancing of sensory input to regions of the brain involved in motor processing, the action output is strengthened by increasing proprioception or body awareness. Proprioception and body awareness is essential in a physical task such as Pilates. Body awareness encompasses a conscious focus and awareness of internal body sensations as well as environmental stimuli. ${ }^{9}$ Body awareness is viewed as a connection between interception and proprioception that enters conscious awareness. It is influenced by previous experience, interpretations, attitudes and affect. Body awareness for movement consists of; the eyes for visualizing movement and coordinating timing, inner 
ear for balance, touch receptors in our skin for feeling and internal sense organs in joints and muscles to feel where your body is in space. Awareness can be enhanced by using one or more of the senses, and further enhanced by ascribing meaning to the goal of the movement through the emotional link. Establishing meaning through an emotional link helps create additional links that enhance learning rather than inhibiting the goal. ${ }^{2}$

Immordino references studies such as ${ }^{10}$ and.${ }^{11}$ Damasio ${ }^{10}$ argues that learning would be physically impossible if a neurological connection was not made between the sensory and motor regions of the brain. These systems of connection were named by the researchers, the convergence zones. In further describing these domains, Damasio ${ }^{11}$ and his colleagues describe this complex system as a conversion between perception and action, based on input from such domains as the visual system, language and social functioning, which can form into a goal directed thought. Immordino additionally references the social learning aspect as described in studies ${ }^{12,13}$ which reveal in part the link between the mirror neuron system and social learning. The studies included within Immordino's examination on learning include the link between the mirror neuron system, as seen as a social gateway to perception of an action, and the emotional component to the mirror neuron system as it relates to internalization of the goals of another's actions, as well as their emotion state surrounding the goal. Emotion is therefore and important component to consider in regards to learning.

\section{Emotional priming}

In a study by Ramachandra et al., ${ }^{14}$ physiological reactions to emotional words were tested in 25 undergraduate student participants. ${ }^{14}$ These participants were comprised of eight men and 17 women with a mean age of 21.5 years. Participants were assessed using galvanic skin response and heart rate monitoring. Researchers presented the participants with a series of stimuli, which consisted of six emotional, digitally recorded vocalizations that representing happiness, sadness, anger, fear, disgust. Participants were also presented with a neutral stimulus. Researchers measured galvanic skin response and heart rate by placing electrodes on each participant's skin. Baseline responses were measured in the participants as each of the six vocalization was given through ear buds. This was followed by a second procedure whereby the participants were asked to consider how they would express their own vocalization for the six emotions. The results indicated an increase in skin reactivity when listening to the vocalizations over that of the base line recordings. However, compared to the neutral vocalization, only fear, anger and sadness elicited a greater skin response. Researchers found that heart rate decreased with the neutral stimuli over that of fear, anger or sadness. Thinking about emotion and listening to emotional stimuli produce similar galvanic and heart rate responses among participants. This may point towards the fact that the mirror neuron system is activated in both thinking about emotion, as one would through empathy, and during vocalization of emotion. The study by Ramachandra ${ }^{14}$ and his colleagues reference recent studies ${ }^{15-18}$ using both neuroimaging and electrophysiological testing procedures, reveal that specialized sets of neurons, specifically the mirror neuron system are implicated not only in the perception and imitation of action, but include cognitive and emotional functions as well as motor functions.

Ramachandra et al., ${ }^{14}$ state that more recent studies ${ }^{19}$ reveal the existence of an auditory mirror neuron system in the premotor cortex, that activates while listening to an action as well as performing the action separately. A prominent link the researchers write, is the connection between the limbic lobe, which processes emotion and the mirror neuron system of the motor and pre-motor cortex. The link the researchers reference is the anterior insula. Ramachandra et al., ${ }^{14}$ report that it is possible to indirectly study the function of the mirror neuron system through psychophysiology measures. Their study uses such techniques to better understand the neural mechanisms of vocal emotions. ${ }^{14}$

Results indicated that the mirror neuron system acts as a pathway between cognitive processing and emotions, language and motor processing. These findings are important as it shows a complete circuit from sensory input to both the cognitive regions of the brain and well the emotional processing regions of the brain, showing the mirror neuron system having a functional role in processing of vocal emotions.

Emotional processing is also linked to motivation. In a study conducted by Custers and Aarts, ${ }^{20}$ researchers demonstrated the role of emotional processing on goal motivation, specifically that positive affect serves as a motivator for a physical goal. Researchers hypothesized that a behavioral goal state, in this case a physical task will be have a more effective outcome when linked with a positive affect over that of negative affect. Throughout a series of six studies researchers illustrated the connection between emotional priming and goal motivation. Emotional priming comprising of positive, negative and neutral verbal cues. Participants were given goal oriented activities, such as completing a puzzle or mouse clicking tasks all associated with a goal outcome. Some groups were given subliminal word priming while completing the task, others groups were given positive verbal cues, such as "doing puzzles is nice".

The groups receiving positive verbal cues were compared against groups receiving negative or neutral cues. The researchers conducted a series of six studies which they linked positive, as well as neutral and negative affect to a behavioral state. The first study was designed to support that linking positive affect to a behavioral state increases the desire of people to want to obtain these goals. The first study consisted of fifty-six undergraduates. The participants worked in separate cubicles. Participants were asked to respond to activities that appeared on the screen, the words represented a mundane activity such as "doing puzzles" or "studying". The participants were asked to give responses at the point when they wanted to engage in the activity.

The participants were shown series of stimulus on a computer screen. These stimulus were paired, a subliminal stimulus or nonword (e.g. MRJLNSLF) followed by either a neutral or positive word on the screen. Following each of the trials, the participants were asked to respond to a question "Do you want to accomplish the activity?" After the six trials, participants were debriefed and checked for the awareness of the subliminal behavioral states. The participants reported no awareness of the behavioral state words, showing that the words were outside of the participants' conscious awareness. The second study was used to rule out alternative explanations, increasing the internal validity of their study. The second study consisted of ninety-three undergraduate students who were randomly assigned to one of three groups: shaping

\section{i. Negative \\ ii. Positive}

\section{iii. Neutral.}

The study procedure was similar to the first study, but the word pairing consisted of a more positive activity statement such as "doing puzzles is nice". Studies three and four were used to explore the 
effort-enhancing effects of linking a positive effect to a behavioral state. In studies three and four the participants were asked to engage in a task. In study three the task such as dot detection was paired with the viewing of subliminal words on the computer screen, consisting of a positive, negative or neutral word. Study four was designed to compare the positively shaped behavioral states in a nonconscious goal versus a conscious goal. The participants in study three were randomly assigned to one of three goal conditions: no goal, unconscious goal, conscious goal. The procedure consisted of the participants partaking in three consecutive tasks: dot-detection, mouse click, number sequence puzzle. At the end of the experiment the participants were instructed that they would be allowed to partake in one additional number-sequence puzzle, if there was sufficient time left. The participants in the conscious goal condition were told following this task "we would appreciate if you will do the numbersequence puzzles". All participants for the last task were asked to take the mouse click task. The speed on the mouse click task served as the dependent variable. The researchers found that linking a goal oriented behavior with a positive affect increased the motivation to obtain the goal, in both conscious and unconscious priming.

Motor processing and emotion is also addressed in a study by Endicott, Johnson, Herring, Hoy and Fitzgerald (2008) in which transcranial magnetic stimulation (TMS) was applied during visual tasks. TMS is a brief pulse delivered to the primary motor cortex regulating nerve conduction related to a motor-evoked potential in a corresponding peripheral muscle. This nerve conduction, or lack of, can be measured via electromyogram. The electromyogram is a device that is used to measure electrical activity in a muscle through an electrode that is attached to the skin of a participant. By the application of TMS researchers could make a correlation between the areas of the brain being stimulated and the nerve conduction in a corresponding muscle group, clarifying the correlation between activities in a particular region of the brain, in this case the mirror neuron system in the motor cortex, and motor activity in peripheral muscles. These techniques were employed during the two visual tasks to show a connection between mirror neuron activation and facial emotion recognition. In this study 20 participants (12 female, 18 male) were given a visual discrimination task, which contained 32 pairs of faces displaying various emotions.

The participants were asked to rate them based on differences, to measure facial emotional processing. A second task was given being a static (image) and dynamic (video) emotional recognition task, whereby the participants were presented with a single facial image and asked to rate it to either fear or surprise. There were 96 random trial with equal numbers of each expression of fear and surprise and equal numbers of static (image) and (dynamic) video representations. The results reflected premotor cortex activation during movement observation, and emotion recognition. This study proposed greater activation of the mirror neuron system in static visualizations, yet the study notes that other studies indicate activation of the system during dynamic visualization as well. This study is important in that the sensory system, in this case the visual system acts as a link to the emotional processing centers of the brain. Emotion linked with sensory input such as vision and touch can further increase receptivity to the motor regions of the brain.

\section{Visual Enhancement of touch}

One technique studied using the visual system in combination with touch is visual enhancement of touch. Visual enhancement of touch is a process whereby sensory input strengthens the learning feedback loop to the areas of the brain responsible for motor action. This is achieved by combining vision and touch, whereby one looks at the area being touched on one's body or viewing someone else touching a particular area of the body. According to a study by Longo et al., ${ }^{21}$ observing one's own body being touched can improve brain reactivity in the area of the body being touched. ${ }^{21}$ This means the person may achieve a better body awareness through sensory proprioception. Participants in this study consisted of 14 volunteers, 13 of which were men between the ages of 19 to $30 y e a r s$ old. Researchers assessed thresholds by delivering pulses of electrical stimulation to the participants' right index finger while the participants engaged in a tactile task. During the experiment, a scalp EEG was used to record activity in the somatosensory regions of the brain. Participants received a series of 1000 pulses delivered to their right median nerve, in between pulses as tactile device introduced a touch, the number of electrical impulses was varied between $30,40,50,60$, or 70 per block, to make the touch stimulation unpredictable. This was carried out while the participant viewed either their own hand in one condition, or an object, the size of their hand in a second condition. Blocks alternated between view hand and view object conditions. EEG data was analyzed following the experiment and the results showed that seeing one's body stimulated motor processing in the primary somatosensory areas of the brain. Data also revealed an increase in tactile acuity while viewing the hand versus the object. These findings substantiate the effectiveness of visual enhancement of touch.

In a study by Hamera ${ }^{22}$ the researcher found that with stimulating the sensory receptors in the skin though touch resulted in activation in the areas of the brain responsible for proprioception in the parietal lobes while also strengthening neural firing. ${ }^{22}$ This finding suggested that that receiving touch can help an individual focus our attention on what muscle to contract or relax. In a comparable study researchers found that neural activity can be triggered when through a brief glimpse of one's own hand being touched. ${ }^{23}$ This finding revealed specialized neural system that creates representations of the body using vision and touch. Thirty-three participants were recruited for this study, 18 of which were female, with a mean age of 24.2. Participants were fitted with scalp EEG electrodes in order to measure responses to stimulation during the experiment. Participants received a series of electrical stimulation signals being delivered to participants' right middle finger. A sensory threshold measurement was created for each individual participant, the mean threshold being $54 \mathrm{~mA}$.

Participants were asked to report either presence or absence of stimulation to the finger. Throughout each trial the participants received either 10 or 20 electrical simulations at 1.4 times that of the mean threshold. During the first trial the participant kept their eyes closed. Then during the second trial, the participants received another 10 to 20 stimulations in the dark, with their eyes open, with a random lighting happening showing either their own hand, or that of an object the size of their hand. During each of the trails the participants received a robotic touch to the right middle finger, at random intervals. Researchers analyzed EEG data following completion of each trial. Results revealed a significant correlation between viewing the hand and sensory response in the brain versus that of viewing the object. This finding suggested that providing even brief visual glimpse of the hand coupled with touch enhanced activation in the somatosensory regions of the brain. This also supported the notion that tactile processing is better activated using vision over that of the participant viewing an object during electrical stimulation. This study demonstrates that the brain creates a more accurate representation of the body, when both vision and touch are utilized, as with visual enhancement of touch. 


\section{Enhanced motor learning for movement practices}

Movement learning involves perception of an action and its goal, imitation of the action and a drive to obtain the goal, which in the proposed study emotion is a component. During movement practices such as Pilates an instructor guides an individual through a learning environment. As previously discussed the brain's receptivity to the environment can have a large impact on the learning outcome. Because of its plasticity, the brain is able to adapt to contextual components and therefore a combination of various techniques can enhance the outcome of motor learning.

Recent researches into athletic practices are currently touching upon the role of the mirror neuron system, motor learning and injury prevention. One such study by Benjaminse and Otten ${ }^{24}$ discuss the role of observational motor learning in sports performance and injury prevention. ${ }^{24}$ The authors report that observational motor learning is the result of the actions of the mirror neuron system and that being they are visuomotor neurons that are activated by both the action being performed, and when a similar action is passively observed, especially when the goal of the action is known. The authors imply that the action of observational learning is a key factor in both athletic performance enhancement and injury prevention. Their rationale is that advancements in motor control knowledge can have implications in both sports performance and injury prevention training. Advancements from studies such as these can be applicable to the rehabilitation of injuries that do occur.

Techniques such as positive emotional priming and visual enhancement of touch applied to motor learning tasks such as Pilates, can allow for the possibility of enhanced motor learning through activation of the regions of the brain responsible for perception, action and execution of movement, in part the mirror neuron system. This added to the brains ability to adapt and change through neuroplasticity, an improved learning environment can be created.

As theorized by Bishop ${ }^{25}$ multi-modal techniques can be applied to movement practices such as Pilates, Yoga, Feldenkrais as well as rehabilitative settings such as physical therapy. Bishops theories cover using what we have discovered through neuroscience, on how motor learning is created, and applies this knowledge to movement based practices. ${ }^{25}$ In her research, she applies brain science to every step of motor learning, for example; Bishop applies internal rehearsal of movement as imagery to enhance activation of the motor cortex and occipital lobes, to create a potential for movement. Bishop applies verbal cues such as action verbs to activate neurons in the temporal region, so that physical movement can be created. She combines vision and touch with a goal oriented movement cue, to enhance the activation of the mirror neuron system. All of her techniques allow for a deeper introspective and exteroceptive learning environment, creating an improved neurological and physical environment for movement learning. Bishop reports that all four lobes of the brain are involved in movement learning, including movement planning in the frontal lobe, feeling and paying attention in the parietal lobes, remembering, hearing and emotions in the temporal lobes, and seeing movement in the occipital lobe. By strengthening the learning environment using techniques to enhance activation in all regions involved in motor learning through enhanced sensory input, one can create improved motor learning. Bishop's ${ }^{25}$ study parallels the theories surrounding the mirror neuron system and mirror box therapy for phantom limb syndrome currently used in physical rehabilitation. If these techniques can assist in better activating the regions of the brain involved in the feedback loop in motor learning, each of these practices and possibly more can benefit from further research in the area of motor learning. ${ }^{25}$
Learning is possible through the malleability of brain structures, this being neuroplasticity. The brain is able to create changes in synaptic strength between neurons and create new circuitry, allowing for learning. In the proposed study it is hypothesized that a group who receiving both the positive emotional priming and the visual enhancement of touch will learn Pilates techniques better and have improved mood and physical self-efficacy scores compared to participants that get regular Pilates training.

\section{Method}

\section{Participants}

An online survey was made from existing and potential clients of Pilates with Anne, Petaluma, California to ask for voluntary participation in the study, an introductory letter was included. Of the population surveyed, a total of 15 people volunteered, of which five have been randomly assigned to a control group. Five participants have been assigned to a regular 1 hour Pilates class 2 times a week for 2 weeks. And five participants have been assigned to a regular Pilates class that includes the independent variables.

\section{Materials}

Standard Pilates spring boards and mats were used in each class with a series of Pilates techniques to be carried out each session as guided by a certified Pilates instructor. This routine was developed according to Joseph Pilates' written instructions as interpreted by Isacowitz. ${ }^{26}$

\section{Physical self-efficacy scale (PS-ES)}

The participants were evaluated using a Physical Self-Efficacy Scale (PS-ES). This scale measures participant's level of physical ability at any given time. This scale consisted of a 22 -item scale that assesses the degree to which respondents have a sense of physical self-efficacy and includes such questions as "I don't feel in control when I take tests involving physical dexterity". ${ }^{5}$ Responses to the questionnaire were rated on a six-point Likert scale, in which 1 reflected "strong agreement" and 6reflected "strong disagreement". Higher rating indicates a higher sense of self efficacy.

\section{Mood survey scale (MSS):}

The Mood Survey Scale (MSS) was used to assess participant's current mood state. Within this measurement scale were two subscales including level of mood (LM) and reactivity to situations (RS). Higher scores reflected higher positive mood states, yet the reactivity to situations a lower score reflects increased physical self-confidence. This scale, which consisted of 19 items measured mood on a continuum ranging from happy to sad. ${ }^{27}$ Example questions included "I generally look at the sunny side of life". Responses were analyzed according to a six-point point Likert scale, in which lower numbers indicated "strong disagreement" while higher numbers indicated "Strong agreement".

\section{Procedure}

Each participant was instructed to complete an on-line PS-ES and MS questionnaire via survey monkey, no more than 8 hours prior to the start of the experiment.

Two of the groups attended separate Pilates classes at the same studio as part of the experimental condition. The participants were taken through a series of standard Pilates techniques. During the first session a Pilates instructor interpretation was completed by the 
researcher who is also a certified Pilates instructor. The observer assessed baseline ability and any change in skill level using a five-point scale. A response of 1 indicated little to no sign of skill acquisition, while a response of 5 indicated a very noticeable or full mastery of acquisition of skill. The research Instructor observed movement techniques at the beginning of the first Pilates session of each of the groups to identify a baseline of physical ability. A second observation was conducted during the last session that occurred two weeks later. No interventions were used during either of the two observations.

The control group did not attend and Pilates classes, were asked to only fill out a PSE and MS on the first day that the other two groups were starting their Pilates classes and on the last day of the experiment. Following the first observation participants in the experimental condition received positive priming cues. These cues consisted of a term from the positive emotional word list, which was then inserted into the verbal cuing for each exercise technique. Example s of verbal cuing questions included "Imagine and Visualize feeling connected with your body, taking pride in your achievement when you feel the mastery of the movement. Cultivate this feeling within yourself". This cueing technique implemented at the start of each exercise demonstration as well as throughout each session. The control groups did not receive positive prime words inserted in their instructed techniques.

Participants assigned to the experimental group also received visual enhancement of touch. During this intervention in the experimental condition, the instructor asked the participant to touch the area of the body needing activation for the movement, and asked the participant to view the area of the body being touched on their own body. The control groups did not receive visual enhancement of touch and the exercises were demonstrated using standard Pilates teaching.

Following these interventions both groups were asked to complete an online version of the PS-ES as well as the MS within eight hours subsequent to their last Pilates session. Participants submitted the questionnaires online.

\section{Results}

Table 1 shows means and standard deviations of skill acquisition including skill change in group 2 (regular Pilates) and group 3 (Pilates with positive emotional cueing and visual enhancement of touch).

Table 2 shows means and standard deviations of physical selfefficacy including sub-scores of physical self-presentation confidence and perceived physical ability and mood scores that also consist of sub-scores of level of mood and reactivity to situations.

Table 3 shows means and standard deviations for Mood Scores, including sub-scores of Level of Mood and Reactivity to situations.

Table I Pre versus post skill acquisition measurements

\begin{tabular}{|c|c|c|c|c|}
\hline & $\begin{array}{l}\text { Group } \\
\text { Pilates }\end{array}$ & 2- Regular & $\begin{array}{l}\text { Group 3- } \\
\text { and VET }\end{array}$ & Pilates with Positive \\
\hline Measure & $M$ & $S D$ & $M$ & $S D$ \\
\hline Pre-Test Skill & 24.75 & 4.57 & 20.75 & 1.26 \\
\hline Post-Test Skill & 30 & 1.41 & 33.50 & 1.91 \\
\hline
\end{tabular}

It was hypothesized that the group receiving positive emotional cuing and visual enhancement of touch would show increases in skill acquisition and improved psychical self-efficacy scores as well as improved mood scores. A comparison was made between the group receiving regular Pilates and Pilates with positive emotional and visual enhancement of touch cuing. Independent samples t-test results showed a statistically significant difference between the preskill mean as reported for group 2, regular Pilates. In group 3 who received positive emotional cueing and visual enhancement of touch, the post-skill mean score was statistically significant over the pre-skill mean. Showing that both groups had a significant increase in skill, with group 3 having a significantly greater increase in skill acquisition (Figure 1) The change in skill mean is reported, again showing a significant increase in skill change of group 3 who received positive emotional cuing and visual enhancement of touch over 2 which was the regular Pilates class where no variables were introduced into the class (Figure 2). Independent samples t-test results in the regular Pilates groups were reported as $t(3.77)=5.25 \mathrm{p}<0.05$, versus the group who received positive emotion cueing and visual enhancement of touch $t(.96)=12.75 \mathrm{p}<0.05$.

Table 2 Physical Self Efficacy scores including sub scores of Physical Self Presentation Confidence, Perceived Physical Ability

\begin{tabular}{llclc}
\hline & \multicolumn{3}{c}{ Group 2-Regular Pilates } & $\begin{array}{l}\text { Group 3-Pilates with } \\
\text { Positive Emotional Cuing } \\
\text { and VET }\end{array}$ \\
\hline Measure & M & SD & M & SD \\
Pre-PSE & 83.75 & 5.90 & 79.25 & 4.11 \\
Post-PSE & 88.5 & 5.25 & 81.00 & 6.22 \\
Pre-PSPC & 47.25 & 4.86 & 43.25 & 5.06 \\
Post-PSPC & 51.00 & 3.16 & 43.25 & 2.50 \\
Pre-PPA & 36.5 & 4.65 & 36.00 & 4.24 \\
Post-PPA & 37.5 & 4.50 & 37.75 & 4.79 \\
\hline
\end{tabular}

Table 3 shows means and standard deviations for Mood Scores, including sub-scores of Level of Mood and Reactivity to situations

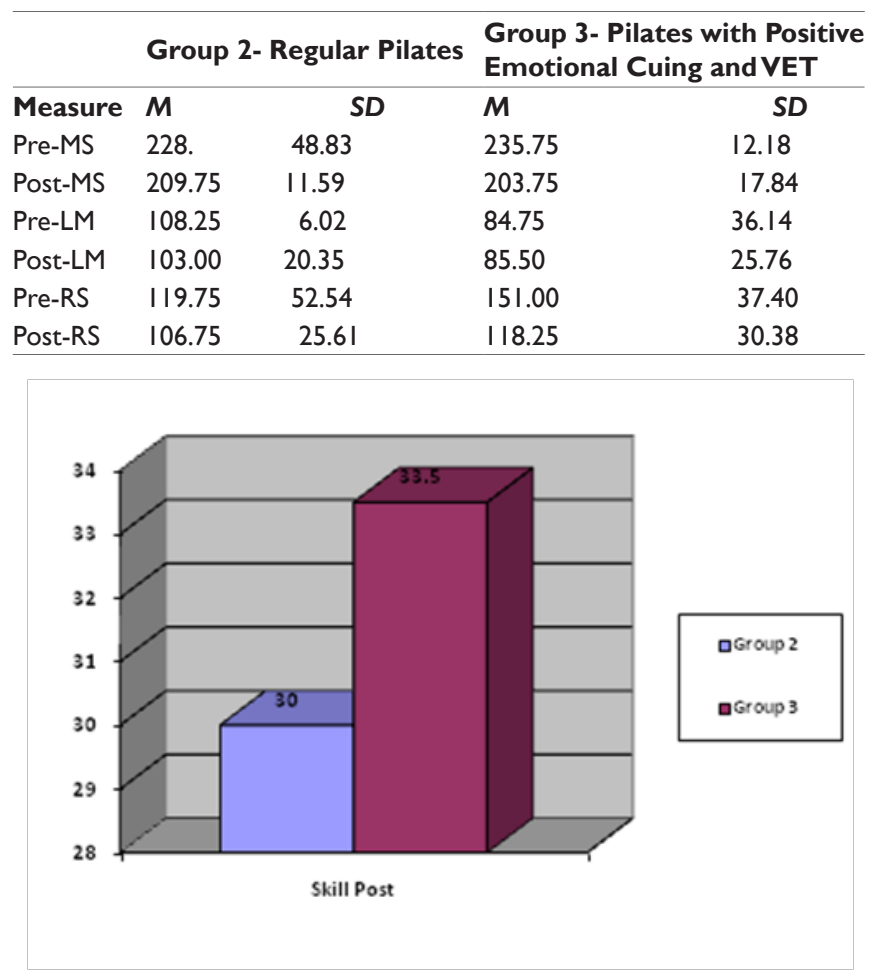

Figure I Post skill.

Group 2=Regular Pilates; Group 3=Pilates with positive emotion andVET.

The hypothesis was supported with these findings of improved skill, especially with group 3 who received positive emotional cuing and visual enhancement of touch during their sessions. 
All three groups were tested on both pre and post physical selfefficacy and mood scores. Group statistics report that groups 1,2 and 3 had increases in mood score and self-efficacy with the greatest change shown in groups 2 and 3 with a significant decrease in the mood sub scale reflecting reactivity to situations. Specifically group 3 showed a greater decrease in reactivity to situations over that of group 2. (Figure 4) This finding is consistent with the hypothesis that with increased physical self-efficacy and increased perceived physical ability scores, scores for reactivity will decrease due to increased confidence in skill acquisition (Figure 3).

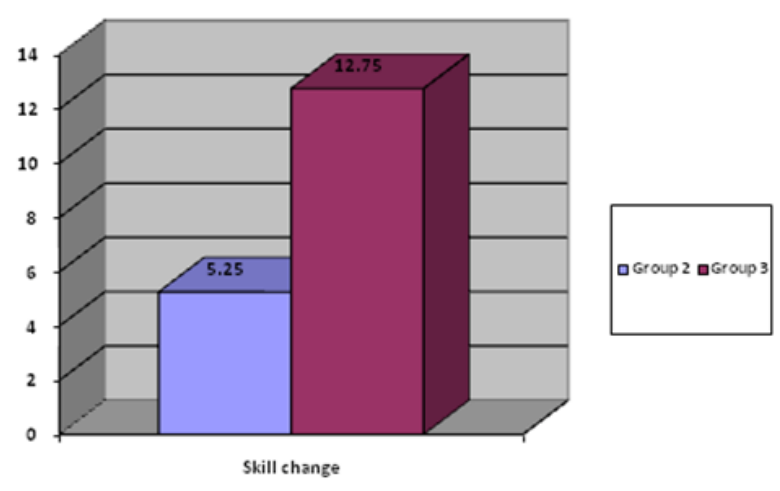

Figure 2 Skill change..

Group 2=Regular Pilates; Group 3=Pilates with positive emotion andVET.

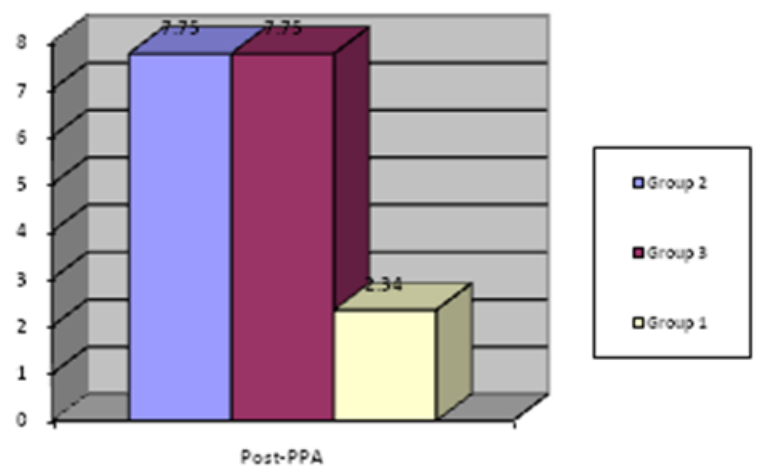

Figure 3 Post- PPA.

Group 2=Regular Pilates; Group 3=Pilates with positive emotion and VET; Group I=control survey only.

Comparing group 2 and 3 in physical self-efficacy, sub scores showed significant difference significance in perceived physical ability (Figure 3).

Overall, the group receiving the on-line survey of physical self-efficacy and mood score showed minimal change. PreMS $m=207, s d=74.97$, Post-MS $m=192.5, s d=68.71$. The groups receiving both regular Pilates and Pilates enhanced with positive mood and visual enhancement of touch showed ranges approaching significance. $t(3)=2.89>0.05$. This difference shows the groups performing exercise had a greater sense of self efficacy over the nonexercise group.

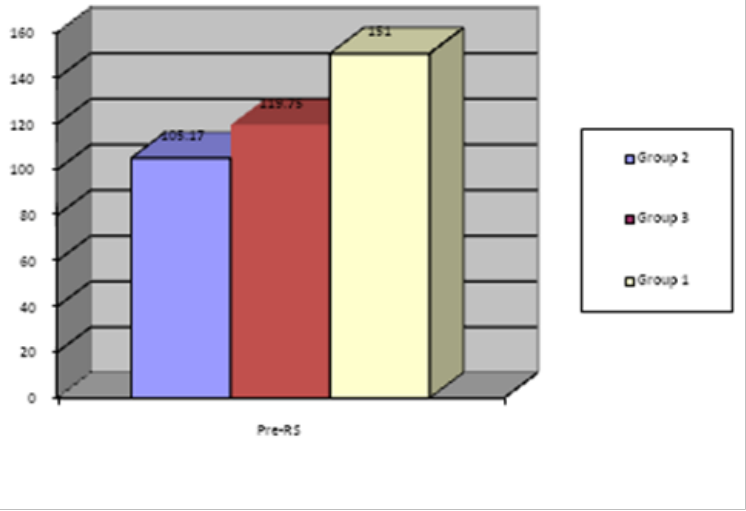

Figure 4 Pre-RS.

Group 2=Regular Pilates; Group 3=Pilates with positive emotion and VET; Group I=control survey only.

\section{Discussion}

It is hypothesized that the group receiving visual enhancement of touch and positive emotional cuing will have increased motor skills in the Pilates tasks as well as have improved mood and self-efficacy scores. This in theory is due to heightened receptivity in the regions of the brain that process motor activity.

In this study, the main hypothesis was supported as the skill levels and physical self-efficacy increased using positive emotional cuing and visual enhancement of touch. Mood score changes also approached significance in both of the Pilates groups where the level of mood and overall mood scores increased, and the reactivity to mood decreased, with greater changes appearing in thegroup that received positive cuing and VET.

Original studies by Rizzolatti \& Craighero $^{28}$ reported that mirror neurons the in brains of macaque monkeys showed activation when the subject watched the action of another monkey bringing food to its mouth or while another monkey was grasping an object. ${ }^{28}$ These early studies uncovered the link between the mirror neuron system and learning. Albert Bandura ${ }^{29}$ developed the theory model that included learning through social imitation, whereby people learn by observation and imitation or modeling and people learn best with a strong sense of self-efficacy. ${ }^{29}$ The current study showed similar results in increased physical self-efficacy following a physical task as in the study by McAuley and Gill ${ }^{4}$ whereby the researchers found that selfconfidence in physical performance could be reliably measured and be a valid instrument of gauging the link between physical performance and physical self-efficacy when the participant had a clear goal. ${ }^{4}$ Likewise, the current study linked the goal with motivation as in the article discussed earlier by Custers and Aarts ${ }^{20}$ whereby emotional processing is also linked to motivation, according to the researchers, the groups receiving positive emotional priming worked harder to achieve the physical goal in their study.

The current study used positive emotional cuing as this goal motivator with results of increased physical self-presentation confidence and increased perceived physical ability scores in the group receiving positive emotional cuing and visual enhancement of touch. This study effectively used variables to enhance a movement learning environment in Pilates, in theory by creating an increase in 
receptivity in the areas of the brain that process motor perception and action.

With advancements in brain imaging techniques, regions of the brain responsible for motor recognition and action have been mapped and more extensive studies have been carried out. This study in combination with brain imaging techniques can potentially further prove that the techniques used in this study are in fact the components that activate motor regions of the brain, as well as show heightened activity in the regions of the brain that process goal motivation and emotions. As brain imaging techniques are refined, the mirror neuron component can be included. Currently a combination of feedback techniques such as fMRI, EEG and TMS are required to pinpoint neural activity.

Researchers such as those discussed who study motor learning report, that with continuing advances in neuroscience, we will be better able to isolate mirror neuron activity and better link their role in movement learning.

It can be reported that the results of this study show that VET was effective in improving physical skill levels, and physical selfefficacy, showing that with increased skill, participants were more self-confident. Over the limited course of time in this study, there may have not been a sufficient amount of time to affect mood scores as greatly as predicted, yet potential was shown as mood scores were approaching significant levels in the group receiving VET and positive emotional cuing.

The small number of participants is one limitation of the current study. It is predicted with a larger number of participants, some of the changes that approach significance would reach significant levels. Therefore, further studies are warranted using larger groups of participants and a model that continues for at least 4-6 weeks. According to Underwood et al., ${ }^{27}$ mood score are most effectively measured over a length of time greater than 4 to 6 weeks. ${ }^{27}$

It also might be a factor to study each component separately. Further studies might group participants into constructs that include just one variable such as visual enhancement of touch, and another group with just positive emotional cuing to do a micro examination of their separate impact on movement learning. Yet in this study, the goal was to show that when skill is positively impacted, mood scores will have a connected improvement.

This study pushes brain research to real world techniques in contexts where teaching experimentation already occurs, such as in a Pilates studio, to see how the neural control of movement studies can be applied to the real world to improve motor learning outcomes.

In summary, brain studies have shown that the pre-motor and parietal regions of the brain involved in action execution are activated when a goal direction action is viewed by the observer. There is increasing evidence that supports that a similar neural mechanism is involved in action imitation that may apply to the domain of sensation, in particular multi modal sensory input. A third factor is the neural structures that tie the goal of an action, the imitation of an action together with emotion and emotions role in goal motivation. Further studies into these mechanisms of learning are forefront in the understanding of the components that can potentially improve a learning environment that contains a motor skill. And with the assistance of brain imaging techniques, studies such as this can prove as an effective study model for continued research in motor learning environments such as physical rehabilitation, competitive sports and possibly somatic based physiological therapies where the main goal is increased physical self-awareness and improved motor learning.

\section{Acknowledgments}

None.

\section{Conflicts of interest}

Author declares there are no conflicts of interest.

\section{Funding}

None.

\section{References}

1. Gallese V, Fadiga L, Fogassi L, et al. Action recognition in the premotorcortex. Brain.1996;119 (Pt 2):593-609.

2. Immordino-Yang MH. The Smoke Around Mirror Neurons: Goals as Sociocultural And Emotional Organizers of Perception and Action in Learning. Mind, Brain, and Education.2008;2(2):67-73.

3. Enticott PG, Johnston PJ, Herring SE, et al. Mirror neuron activation is associated with facial emotional processing. Neuropsychologia. 2008;46(11):2851-2854.

4. McAuley E \& Gill DL. Reliability and validity of the Physical SelfEfficacy Scale in a competitive sport setting. Journal of Sport \& Exercise Psychology. 1983;5:410-418.

5. Ryckman RM, Robbins MA, Thornton B, et al. Development and validation of a physical self-efficacy scale. Journal of Personality and Social Psychology. 1982;42(5):891-900.

6. Medina J. Brain Rules. Pear Press, Seattle WA, USA. 2008

7. Bastiaansen J, Thioux M, Keysers C. Evidence for mirror systems in emotions. Philos Trans R Soc. 2009;364(1528):2391-2404.

8. Keysers C, Wicker B, Gazzola V, et al. A touchingsight: SII/PV activation during the observation and experience of touch. Neuron. 2004;42(2):335-346.

9. Mehling W, Wrubel J, Daubenmier J, et al. Body Awareness: a phenomenological inquiry into the common ground of mind-body therapies. Philos Ethics Humanit Med. 2011;6(6):747-5341.

10. Damasio AR. Time-locked multiregional retroactivation: A systemslevel proposal for the neural substrates of recall and recognition. Cognition. 1989;33(1-2):25-62.

11. Damasio AR, Damasio H. Cortical systems underlying knowledge retrieval: Evidence from human lesion studies. In: Poggio TA \& Glaser DA (Eds.), Exploring brain functions: Models in neuroscience. Wiley, New York, USA. 1993. p.233-248.

12. Wood JN, Glynn DD, Phillips BC, et al. The perception of rational, goaldirected action in nonhuman primates. Science. 2007;317:1402-140.

13. Oberman LM, Pineda JA Ramachandra VS. The human mirror neuronsystem: A link between action observation and social skills. Social Cogn Affect Neurosci. 2007;2(1):62-66.

14. Ramachandra V, Depalma N, Lisiewski S. The role of mirror neurons in Processing vocal emotions: Evidence from psychophysiological data. International Journal of Neuroscience. 2009;119(5):681-690.

15. Bertenthal BI, Longo MR, Kosobud A. Imitative response tendencies following observation of meaningless actions. $J$ Exp Psychol Hum Percept Perform. 2006;32(2):210-225.

16. Decety J, Sommerville J A. Shared representations between self and other; A social cognitive neuroscience view. Trends Cogn Sci. 2003;7:527-533.

17. Fadiga L, Foggasi I, Pavesi G, et al. Motor facilitation during action observation: A magnetic simulation study. J Neurophysiol. 1995;73(6):2608-2611. 
18. Iaocoboni M, Woods RP, Brass M, et al. Cortical mechanisms of human imitation. Science. 1999;286(5449):2526-2528.

19. Gazzola V, Aziz-Zadeh L, Keysers. Empathy and the somatotopic auditory mirrorsystem in humans. Curr Biol. 2006;16(18):1-6.

20. Custers R \& Aarts H. Positive affect as implicit motivator: On the nonconsciousoperation of behavioral goals.J Pers Soc Psychol. 2005;89(2):129-142.

21. Longo M, Pernigo S, Haggard P. Vision of the body modulates processing inprimary somatosensory cortex.Neuroscience Letters. 2011;489(3):159-163.

22. Hamera J. Dancing communities: Performance, difference and connection in the global city. Palgrave Macmillian, Basingstoke, New York, USA. 2007

23. Cardini F, Longo M, Driver J, et al. Rapid enhancement of touch from non-informative vision of the hand. Neuropsychologia. 2012 50(8):1954-1960
24. Benjaminse A, Otten E. ACL injury prevention, more effective with a different way of motor learning? Knee Surg Sports Traumatol Arthrosc. $2011 ; 19(4): 622-627$.

25. Bishop CA. Cognitive neuroscience in mind body education: Teaching to feel enhances learning to move. 2011.

26. Isacowitz R. Pilates. Human Kinetics, Champaign IL, USA. 2006.

27. Underwood B, Froming WJ. The mood survey: A personality measure of happy and sad moods. Journal of personality Assessment .1980;44(4): 404-414.

28. Rizzolatti G, Craighero. The mirror neuron system. Annu Rev Neurosci. 2004;27:169-192.

29. Bandura A. Social learning theory. Prentice Hall, Englewood Cliffs NJ, USA. 1977. 